\title{
Throughput Maximization of Cognitive Radio Multi Relay Network with Interference Management
}

\author{
Pradip Varade ${ }^{1}$, Akanksha Wabale ${ }^{2}$, Ravinder Yerram ${ }^{3}$, Rupesh Jaiswal ${ }^{4}$ \\ ${ }^{1}$ Department of E\&TC, Rajshree Shahu College of Engineering, SPPU, Pune, India \\ ${ }^{2,3,4}$ Department of E\&TC, Pune Institute of Computer Technology, Pune, India
}

\section{Article Info \\ Article history: \\ Received Dec 14, 2017 \\ Revised Jan 3, 2018 \\ Accepted Mar 29, 2018}

Keyword:

Cognitive radio

Cognitive relay networks

Interference management

Jain's fairness index

OFDM

Resource allocation

\begin{abstract}
In this paper, an Orthogonal Frequency Division Multiplexing (OFDM) based cognitive multi relay network is investigated to maximize the transmission rate of the cognitive radio (CR) with enhanced fairness among CR users with interference to the primary users (PUs) being managed below a certain threshold level. In order to improve the transmission rate of the CR, optimization of the subcarrier pairing and power allocation is to be carried out simultaneously. Firstly joint optimization problem is formulated and Composite Genetic and Ordered Subcarrier Pairing (CGOSP) algorithm is proposed to solve the problem. The motivation behind merging genetic and OSP algorithm is to reduce the complexity of Genetic Algorithm (GA). Further, to have a fair allocation of resources among CR users, the Round Robin allocation method is adopted so as to allocate subcarrier pairs to relays efficiently. The degree of fairness of the system is calculated using Jain's Fairness Index (JFI). Simulation results demonstrate the significant improvement in transmission rate of the $\mathrm{CR}$, low computational complexity and enhanced fairness.
\end{abstract}

Copyright $\odot 2018$ Institute of Advanced Engineering and Science. All rights reserved.

\section{Corresponding Author:}

Pradip S. Varade,

Department of Electronics and Telecommunications Engineering,

Rajshree Shahu College of Engineering,

Savitribai Phule Pune Univaersity,

Pune, India 411046.

Email: pradip_varade@rediffmail.com

\section{INTRODUCTION}

In wireless communication, radio spectrum is one of the scarcest resources. According to the Federal Communications Commission (FCC), approximately $85 \%$ of the times licensed frequency bands are unused, and thus wasted [1]. Hence, to make efficient spectrum usage cognitive radio concept is discussed where unlicensed users can dynamically use the spectrum bands of the licensed users in an opportunistic way [2]. Various spectrum sensing methods particularly hybrid sensing method is discussed in [3]. To improve channel efficiency in CRN adaptation of optimal weight with various diversity techniques is proposed [4]. To address the problem of delay in CR transmission data cascading scheme is proposed wherein CR information is stored in between nodes [5].The CR users can improve their transmission rate with the help of relay-based network by relaying their messages through a number of relays. Various relaying methods such as Decode-and-Forward (DF) and Amplify-and-Forward (AF) have been discussed in [6]. An OFDM-based relay-aided communication system is a promising technique to improve the transmission rate of the CR users.

Previously, several studies have been done to improve the capacity of OFDM-based cognitive relay network. The capacity of the relay network is improved by subcarrier pairing and power allocation with and without interference management [7]-[11]. In [10]-[12], an ordered subcarrier pairing (OSP) scheme has been 
used for subcarrier pairing. However, in [10], optimal power allocation is not considered. As a solution, classic water-filling method has been used for power allocation, which is not optimal for cognitive environment, as it requires power allocation with interference management. In [11], subcarrier pairing and power allocation problem of OFDM-based relay network is optimized with interference management using a GA framework with the DF relaying scheme.

In [13], OFDM-based CR system is considered, and a significant gain in terms of the total transmission rate is achieved over the underlay and overlay spectrum access mechanism without considering relay network. In [14], a CR decision engine is proposed, which determines the optimal radio transmission parameters using GA for optimal spectrum allocation in CR. The resource allocation in OFDM based cognitive radio relay networks $(\mathrm{CRRN})$ is carried out with interference to the PUs is managed below some threshold level using Heterogeneous Genetic Algorithm (HGA) to achieve optimum transmission rate [15].

All the aforementioned work carried out for resource allocation in OFDM based CRRN to the best of our knowledge is based on the assumption that single relay is able to relay the signals from all subcarriers of the available spectrum band; ignoring the practical possibility. Furthermore fairness in allocating resources among CRs in OFDM based CRRN is not addressed which is highly required for guaranteeing quality of service (QoS). In this paper the resource allocation problem in OFDM based CRRN with multiple relays is investigated to maximize the transmission rate of the CR. These relays will help CR users to transmit their signals to their intended destination efficiently proving more practical approach. Multiple relays can efficiently relay signals compared to one single relay in the system as used in [15]. Though multiple relay systems are used in [6], [9], they have not considered in the cognitive radio environment, which has been done in this paper. Firstly joint optimization problem is formulated and Composite Genetic and Ordered Subcarrier Pairing (CGOSP) algorithm is proposed to solve the problem. The novel approach of combining GA and OSP algorithm, i.e. CGOSP gives a noticeable improvement in the transmission rate of the CR users where the genetic approach is used for optimal power allocation and OSP is used for subcarrier pairing. This algorithm reduces the complexity of HGA algorithm, and it also improves the transmission rate of the CR users by maintaining interference to PUs below the threshold.

In order to guarantee QoS requirements of CR users fair allocation of the resources to these users, are carried out by allocating subcarrier pair groups to relays and then these relays to the CR users efficiently. A novel Round Robin allocation technique is proposed to allocate subcarrier pair group to relays to enhance degree of fairness which is measured using JFI. The rest of this paper is organized as follows: Section 2 presents the system model and problem formulation. The CGOSP algorithm is explained in Section 3. Simulation results are presented in Section 4. Section 5 concludes the paper.

\section{SYSTEM MODEL AND PROBLEM FORMULATION}

As shown in Figure 1, a two-hop OFDM-based cognitive relay network system is considered. This system consists of two transmitter-receiver pairs of the CR users, $\mathrm{N}$ dedicated relays and M transmitterreceiver pairs of the PUs. Relays are assumed to be operated with Decode and forward (DF) protocol. The transmission of CR users takes place in two phases. In the first phase, CR sources transmit the messages to the relays and then all the relays decode the received messages. In the second phase, the relays again reencode the messages and transmit them to the destination. Here, we assume that the allocated spectrum band is divided into two $\mathrm{K}$ number subcarriers. The first $\mathrm{K}$ subcarriers are the allocated to sources and the other $\mathrm{K}$ subcarriers are allocated to the relays. As the whole transmission of the CR users occurs through relays, we formulate pairs of the subcarriers. If the transmission takes place on the $\mathrm{i}^{\text {th }}$ subcarrier in the first phase and on the $\mathrm{j}^{\text {th }}$ subcarrier in the second phase then $(\mathrm{i}, \mathrm{j})$ is said to be the subcarrier pair [15]. Our aim is to form such subcarrier pairs and allocate powers to them in order to improve the transmission rate of the CR users. However, the interference from these subcarriers to the PUs must be below the predefined threshold level and fair allocation of the resources among CR users by maintaining JFI [16] above $\mathbf{J}_{\text {min }}$. The signal received on one of the relays on the $\mathrm{i}^{\text {th }}$ subcarrier can be expressed as,

$$
y_{i}^{R}=\sqrt{p_{i}^{S}} H_{i}^{S R} d_{i}+I_{i}^{P R}+N_{0}
$$

where, $\mathrm{i} \in\{1,2, \ldots, \mathrm{K}\}, p_{i}^{S}$ is the power of CR's normalized signal $d_{i}, H_{i}^{S R}$ is a complex channel coefficient from CR to relay, $I_{i}^{P R}$ is an interference introduced by PU to the relay and $N_{0}$ is additive white Gaussian noise with zero mean and unit variance. Similarly, the signal received at CR destination can be expressed as,

$$
y_{j}^{D}=\sqrt{p_{j}^{R}} H_{j}^{R D} r_{j}+I_{j}^{P D}+N_{0}
$$


where, $\mathrm{j} \in\{1,2, \ldots, \mathrm{K}\}, \mathrm{p}_{\mathrm{j}}^{\mathrm{R}}$ is the power of relay's normalized re-encoded signal, $r_{j} . H_{j}^{R D}$ is a complex channel coefficient from relay to the destination and $I_{j}^{R D}$ is an interference introduced by PU to the destination.

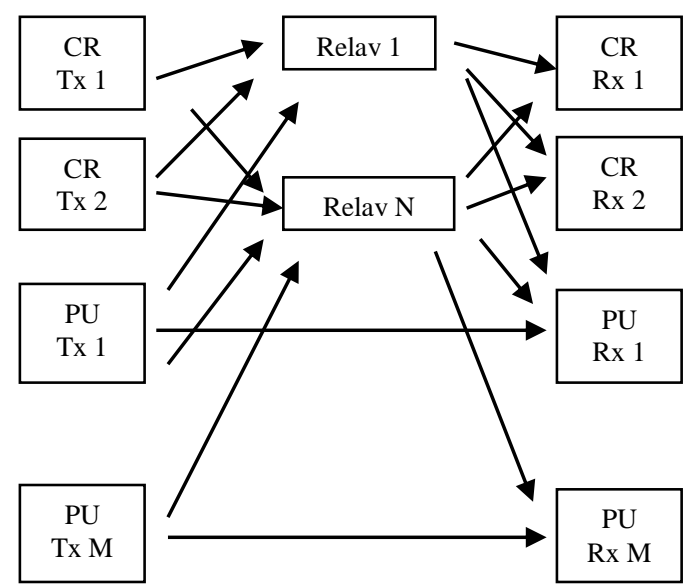

Figure 1. Cognitive radio multi relay network mode

The allocated transmission rate of the CR users must be

$$
R(i, j)=\min \left\{\log \left(1+\alpha_{i}^{S R} p_{i}^{S}\right), \log \left(1+\alpha_{j}^{R D} p_{j}^{R}\right)\right\}
$$

where, $\alpha_{i}^{S R}$ and $\alpha_{j}^{R D}$ are normalized channel gain at relay and destination respectively. We have assumed that the whole spectrum is shared by M PUs. Hence, an interference introduced to $\mathrm{m}^{\text {th }} \mathrm{PU}$ can be given by,

$$
\sum_{i=1}^{K} p_{i}^{S} \Psi_{i}^{S, m} \quad \text { and } \quad \sum_{i=1}^{K} p_{j}^{R} \Psi_{j}^{R, m}
$$

These are the interferences from the CR sources and relays respectively. Here $\Psi_{i}^{S, m}$ is an equivalent channel gain from $\mathrm{i}^{\text {th }}$ subcarrier to the $\mathrm{m}^{\text {th }} \mathrm{PU}$ and $\Psi_{j}^{R, m}$ is an equivalent channel gain from $\mathrm{j}^{\text {th }}$ subcarrier to the $\mathrm{m}^{\text {th }} \mathrm{PU}, \mathrm{m} \in\{1,2, \ldots \mathrm{M}\}$. These two interferences must be below a certain threshold level.

\subsection{Problem formulation}

For the proposed system model discussed in the previous section, the aim is to maximize the overall transmission rate over all the subcarriers for the CR users by keeping the interference to PU below predefined threshold. Let $I(i, j)$ be the indicator for subcarrier pairing. This indicator has value 1 if the $i^{\text {th }}$ subcarrier is paired with the $\mathrm{j}^{\text {th }}$ subcarrier, otherwise it is zero. Let ' $\mathrm{N}$ ' be the number of relays in the network, and each relay can relay only ' $L$ ' number of subcarrier pairs. Let ' $U$ ' $(n, i, j)$ be the indicator for relay selection. The value of this indicator will be 1 if the $n^{\text {th }}$ relay is used for the subcarrier pair $(i, j)$, otherwise it is zero. The overall optimization problem can be written as,

$$
\begin{aligned}
& R=\max _{p_{i}^{S}, p_{j}^{R}, I(i, j), U(n, i, j)} \sum_{n=1}^{N} \sum_{i=1}^{K} \sum_{j=1}^{K} U(n . i . j) I(i, j) R(i, j) \\
& C 1: p_{i}^{S} \geq 0, p_{j}^{R} \geq 0, \forall i \in\{1,2, \ldots \ldots, K\} \text { and } j \in\{1,2, \ldots \ldots, K\} \\
& C 2: \sum_{i=1}^{k} p_{i}^{S} \Psi_{i}^{S, m} \leq P_{\text {thresh }} \forall m \in\{1,2, \ldots \ldots M\} \\
& \sum_{i=1}^{k} p_{j}^{R} \Psi_{j}^{R, m} \leq P_{\text {thresh }} \forall m \in\{1,2, \ldots \ldots M\} \\
& C 3: \sum_{i=1}^{K} p_{i}^{S} \leq P_{S} \text { and } \sum_{j=1}^{K} p_{j}^{R} \leq P_{R} \\
& C 4: \sum_{i=1}^{K} I(i, j)=1 \quad \forall j \text { and } \sum_{j=1}^{K} I(i, j)=1 \forall i
\end{aligned}
$$




$$
\begin{aligned}
& I(i, j) \in\{0,1\}, \quad \forall(i, j) \\
& C 5: \sum_{n=1}^{N} U(n, i, j)=1, \quad \forall(i, j) \\
& \sum_{i=1}^{K} \sum_{j=1}^{K} U(n, i, j)=L, \quad \forall n
\end{aligned}
$$

Target function in Equation (5) is the transmission rate over all the subcarrier pairs used by the CR users and relay. Our aim is to maximize this target function simultaneously satisfying all the constraint equations that are mentioned from Equations (6) to (13). First constraint equation states that the power allocated to each subcarrier on both source and relay side must be greater or equal to zero. Equation (7) ensures that the interference due to the subcarriers used by the CR transmitters to the primary receivers must be below the predefined level $\mathrm{P}_{\text {thresh }}$. Equation (8) also ensures the same, but for the subcarriers used by various relays. Equation (9) corresponds to the total power, which can be used for the CR source and relay respectively. The constraints Equation (10) and Equation (11) imply that each subcarrier of the CR and relay can only be used once. Equation (12) and Equation (13) give constraint to relay. It shows that every subcarrier pair can be relayed only through one relay, and each relay can provide service to L number of subcarrier pairs. Allocation of subcarrier pairs to the relays and relays to the two CR users:

Transmission rate achieved by CR_1:

$$
\mathrm{R}(1)=\sum_{\mathrm{n}=1}^{\mathrm{N} / 2} \sum_{\mathrm{i}=1}^{\mathrm{K}} \sum_{\mathrm{j}=1}^{\mathrm{K}} \mathrm{U}(\mathrm{n} . \mathrm{i} . \mathrm{j}) \mathrm{I}(\mathrm{i}, \mathrm{j}) \mathrm{R}(\mathrm{i}, \mathrm{j})
$$

Transmission rate achieved by CR_2:

$$
R(2)=\sum_{n=\frac{N}{2}+1}^{N} \sum_{i=1}^{K} \sum_{j=1}^{K} U(n . i . j) I(i, j) R(i, j)
$$

Such that $R=R(1)+R(2)$ ter subcarrier pairing and allocation of power to these subcarrier pairs, to the relays and then these relays to the CR users such that fairness is achieved and JFI can be calculated as follows and must be maintained above the Jain's index threshold $\mathrm{J}_{\min }$.

$$
\mathrm{JFI}=\frac{\left|\sum_{\mathrm{i}=1}^{2} \mathrm{R}(\mathrm{i})\right|^{2}}{2 \sum_{\mathrm{i}=1}^{2} \mathrm{R}(\mathrm{i})^{2}} \geq \mathrm{J}_{\min }
$$

\subsection{Implementation details}

Subcarrier pairing, power allocation and fair allocation of resources to SUs are the three essential parts of our optimization problem. In the proposed CGOSP algorithm, two algorithms are merged. One is the GA for power allocation part [15], [17] and other is an OSP algorithm for subcarrier pairing [10] unlike where GA is used for both subcarrier pairing and power allocation parts [15]. Details of CGOSP algorithm are given from Step 1 to Step 6.

Step 1: OSP for subcarrier pairing

As given in [10], the two subcarriers; one from the source to relay and other from the relay to the destination, are paired according to their actual channel strengths; first, the subcarriers on both the sides - on the source side and on the relay side, are sorted according to their channel strengths.

Step 2: Initialization for power allocation

Population initialization for power allocation i.e., $\mathrm{p}_{\mathrm{i}}^{\mathrm{S}}$ and $\mathrm{p}_{j}^{\mathrm{R}}$ can be done first by solving Lagrange dual problem and then applying KKT residue method [15]. This will reduce the number of iterations required to converge the algorithm to an optimal solution.

Step 3: Evaluation

The fitness value of each parent chromosome in an initialized population is calculated using fitness function. Fitness function is given by Equation (5) without the relay constraint term $U$ (n, i, j). By substituting value for $\mathrm{I}(\mathrm{i}, \mathrm{j}$ ) obtained in step 1 in Equation (5), fitness value of each parent chromosome can be calculated.

Step 4: Selection

Parent chromosomes giving maximum fitness value are retained and rest of the chromosomes are discarded. Selected chromosomes undergo crossover and mutation to generate offsprings.

Step 5: Crossover

The crossover operation in the power allocation part is based on the arithmetic crossover operation. For arithmetic crossover, first mutation mask is created using random sequence of zeros and ones. This random sequence is generated with an equal probability generation of zero and one. This mutation mask is

Throughput Maximization of Cognitive Radio Multi Relay Network with Interference ... (Pradip Varade) 
created for all the subcarriers i.e., either overlapped with the PU's band or not. If the element in the mask is 1 then the crossover is done using following equations.

$$
\mathrm{P}_{\mathrm{a}^{\prime}}^{\mathrm{S}}=(1-\beta) * \mathrm{P}_{\mathrm{a}}^{\mathrm{S}}+\beta *
$$

and

$$
\mathrm{P}_{\mathrm{b}^{\prime}}^{\mathrm{S}}=\beta * \mathrm{P}_{\mathrm{a}}^{\mathrm{S}}+(1-\beta) * \mathrm{P}_{\mathrm{b}}^{\mathrm{S}}
$$

where, $\mathrm{P}_{\mathrm{a}}^{\mathrm{S}}$ and $\mathrm{P}_{\mathrm{b}}^{\mathrm{S}}$ are the corresponding gene values (power allocation) of the two parent chromosomes. $\mathrm{P}_{\mathrm{a}^{\prime}}^{\mathrm{S}}$ and $\mathrm{P}_{\mathrm{b}^{\prime}}$, are the corresponding gene values (power allocation) of the two offspring chromosomes and $\beta$ is randomly distributed in $(0,1)[15]$.

Step 6: Mutation

For uniform mutation, first subcarriers are divided into two parts: the subcarriers, which are overlapped with the PUs, and the subcarriers, which are not overlapped with the primary band. Mutation mask is generated according to mutation probability $\mathrm{pm}(0<\mathrm{pm}<1)$. If the mutation mask of the corresponding element is one then any gene value is selected for mutation [15]. The CGOSP algorithm ends here with an optimization of the subcarrier pairing and power allocation.

Step 7: Resource allocation

The given $\mathrm{K}$ subcarrier pairs are relayed through $\mathrm{N}$ relays. To achieve the fairness in resource allocation, two things are needed to optimize first is to form $\mathrm{N}$ groups of the subcarrier pairs and then decide which group is relayed through which particular relay. Second is allocation of these relays to the CR use. Firstly, the subcarrier pairs are sorted according to their signal-to-noise ratio (SNR). In an ordered allocation of the subcarrier pairs, first L numbers of the subcarriers with maximum SNR are assigned to relay 1 and then the next L number of subcarriers with maximum SNR to relay 2 and so on. Hence, if we allocate first half relays to first CR users and another half to second then the transmission rate achieved by the first CR user will be more, and this will reduce JFI.

To improve the JFI factor, there should be fair allocation of the subcarriers to all the relays, and fair allocation of these relays to the CR users. For this purpose, Round Robin allocation of the subcarriers to the relays is adopted. Here sorting of the subcarrier pairs according to their corresponding SNR is carried out, then allocation of the subcarrier pair with highest SNR value to the first relay, then second highest to the second relay, and so on. Further, allocate $(\mathrm{N}+1)$ the subcarrier pair to the first relay and $(\mathrm{N}+2)$ th subcarrier pair to the second relay, and so on in a round robin fashion. After that, allocate first half relays to the first CR user and the remaining relays to the second CR user. With this Round Robin allocation method, each CR user will achieve almost similar transmission rates, and our system will be fairer. The steps used in proposed CGOSP algorithm is summarized in Table 1. Algorithm divides in two phases one is implementation of CGOSP algorithm and second is resource allocation to enhance fairness and compute JFI.

\begin{tabular}{|c|c|}
\hline Input & $d_{i}, H_{i}^{S R}, I_{i}^{P R}, N_{0}, r_{j}, H_{j}^{R D}, I_{j}^{R D}, \alpha_{i}^{S R}, \alpha_{j}^{R D}$ \\
\hline Output & $I(I, j), p_{i}^{S}, p_{j}^{R}, R, J F I$ \\
\hline \multirow[t]{16}{*}{ Phase I } & Implementation of CGOSP algorithm \\
\hline & Case 1: OSP algorithm for subcarrier pairing \\
\hline & $\begin{array}{l}\text { i) Sort the subcarriers on the source and relay side according to their channel gains } \\
\text { ii) Assign index from } 1 \text { to } \mathrm{K} \text { to these sorted channels. }\end{array}$ \\
\hline & iii) Form subcarrier pairs by pairing subcarriers with same index i.e.I (i, j) \\
\hline & Case 2: GA for power allocation \\
\hline & Initialization: Create parent chromosomes using the methods in [17]. \\
\hline & While (all constraint equations are not satisfied) \{ \\
\hline & i) Compute the fitness values of all the parent chromosomes using Equation (V) \\
\hline & ii) Select the parent chromosomes with highest fitness value. \\
\hline & iii) Apply arithmetic-based crossover on the selected chromosomes [15]. \\
\hline & iv) Apply uniform mutation on the crossovered chromosome [15]. \\
\hline & v) Save this new chromosome as parent chromosome. \\
\hline & vi) Compute the values of all constraint equations. $\}$ \\
\hline & Get chromosome with the maximum fitness value and return $p_{i}^{S}, p_{j}^{R}$ And I (I. j). \\
\hline & Resource allocation to enhance fairness \\
\hline & i) Apply Round Robin allocation method using Equation (14 ) and (15) \\
\hline Phase II & ii) Calculate JFI using Equation (16) and measure the degree of fairness. \\
\hline
\end{tabular}

Table 1. CGOSP Algorithm and Implementation Details 


\section{SIMULATION RESULTS}

Extensive simulations are carried out to compare the performance of our proposed algorithms with existing works in terms of transmission rate. Here, OFDM-based cognitive relay environment is consider as explain in the Section 2. The channel coefficients $\alpha_{i}^{S R}$ and $\alpha_{j}^{R D}$ of the subcarriers for source to relay and relay to destination links are generated using methods in [6]. The variance of the additive noise $N_{0}$ is assumed to be unity. Equivalent interfering channel gains $\Psi_{i}^{S, m}$ and $\Psi_{j}^{R, m}$ can be generated from the methods discussed in [18]. One more assumption is that the power requirement is same for source and all the relays. Also, for relay-constrained algorithms, the power $P_{R}$ is divided equally and allocated to all the relays in relayconstraint algorithms before power allocation. The proposed CGOSP algorithm is implemented with and without relay constraint. To compare results the OSP method [11] is designed without power allocation while the HGA method [15] is designed for power allocation with and without relay constraint.

For implementing various algorithms discussed the number of subcarriers used are $\mathrm{K}=32$ and $\mathrm{K}=64$. The number of PUs are M=3. For relay-constraint HGA and CGOSP algorithm, we use the number of relays $\mathrm{N}=4$, and $\mathrm{N}=8$ for $\mathrm{K}=32$ and $\mathrm{K}=64$ respectively, each relay can relay max $\mathrm{L}=8$ numbers of subcarrier pairs. For OSP, simple cooperative network with one relay, which can serve all the subcarriers in the network, is considered, with equal power allocated to all the subcarriers. Practically, we cannot apply equal power to all the subcarriers because it will increase the interference. The GA is implemented with ordered subcarrier pairing and optimal power allocation, and with interference management constraint $P_{\text {thresh }}=-10 \mathrm{~dB}[15]$.

It is observed from Figure 2 and Figure 3 comparing all the algorithms in terms overall transmission rate for the same range of transmitting power with $K=32$ and $K=64$ respectively. The transmission rate of the proposed CGOSP algorithm without relay constraint outperforms over all algorithms. The relay-constraint CGOSP algorithm has more transmission rate compared to the other three algorithms. Figure 2 and Figure 3 show that the proposed CGOSP algorithm, improves the transmission rate by about $9.08 \%$ and $12.96 \%$ respectively without imposing relay constraints. However, even if we put relay constraint, CGOSP algorithm has transmission rate improvement of about $6.13 \%$ and $11.28 \%$ respectively. Ultimately, with relay constraint on both GA and CGOSP algorithm, CGOSP algorithm outperforms, and it shows transmission rate improvement of about $17.88 \%$ and $11.22 \%$. Figure 3 depicts similar performance with enhanced transmission rate as compared to Figure 2 due increased subcarriers. For instance the transmission rate for CGOSP algorithm increased by $54 \%$.

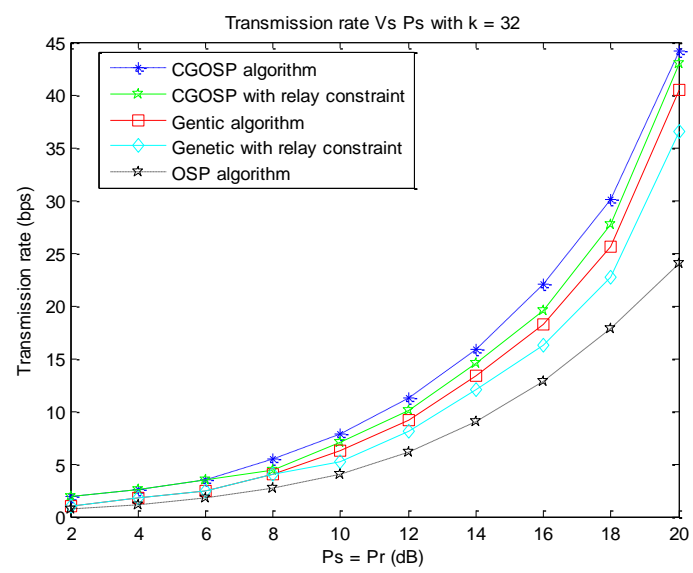

Figure 2. Transmission rate comparison for various algorithms with $\mathrm{K}=32, \mathrm{M}=3, \mathrm{P}_{\text {thresh }}=-10 \mathrm{~dB}$ and $\mathrm{N}=4$

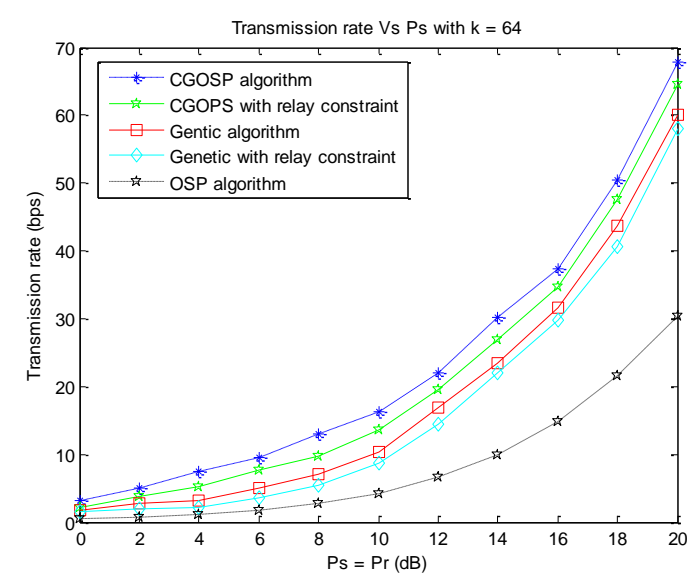

Figure 3. Transmission rate comparison for various algorithms with $\mathrm{K}=64, \mathrm{M}=3, \mathrm{P}_{\text {thresh }}=-10 \mathrm{~dB}$ and $\mathrm{N}=8$

Figure 4 and Figure 5 shows the variation in the sum rate with change in the number of primary users, keeping power constraint $20 \mathrm{~dB}$ and interference management constraint $-10 \mathrm{~dB}$ with $\mathrm{K}=32$ and $\mathrm{K}=64$ respectively. It is evident from Figure 4 and Figure 5, a drastic decrease in the transmission rate for GA with and without relay constraint because the corresponding subcarrier pairing method is not yielding good results. CGOSP has better transmission rates than GA and GA with relay constraint [15]. The transmission rates of all algorithms reduces with the increasing number of PUs. Transmission rate severely drops after number of $\mathrm{PU}=4$ as more number of PU introduces large interference to SU. 


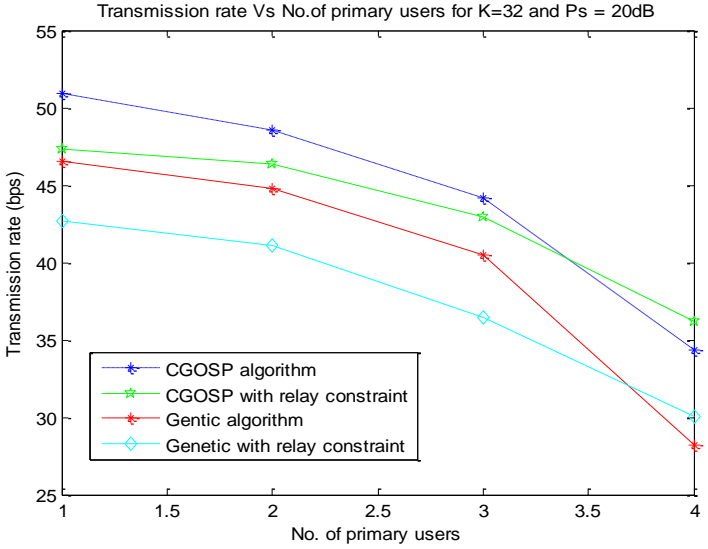

Figure 4. Transmission rate versus number of primary users with $\mathrm{K}=32, \mathrm{~N}=4$ and $\mathrm{P}_{\text {thresh }}=-10 \mathrm{~dB}$

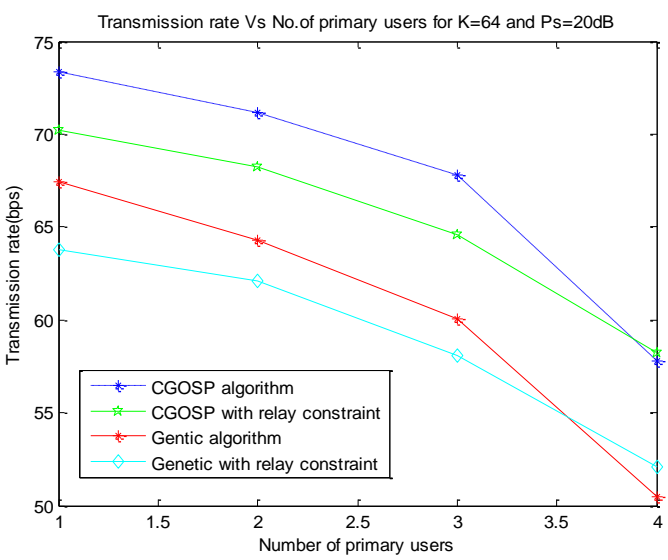

Figure 5.Transmission rate versus number of primary users with $\mathrm{K}=64, \mathrm{~N}=8$ and $\mathrm{P}_{\text {thresh }}=-10 \mathrm{~dB}$

In Figure 6 and Figure 7, we vary the number of relays to see the effect of changing the number of relays on the transmission rate of the $\mathrm{CR}$ users with maximum power constraint on the source and relay are $20 \mathrm{~dB}$ and $\mathrm{K}=32$ and $\mathrm{K}=64$ respectively. From Figure 6 and Figure 7, it is evident that transmission rate increases with increase in number of relays however CGOSP algorithm offers superior performance. In both the graphs, it is observed that initially there is a drastic increase in the transmission rate as number of relays has increased. However, after that, the rate of increase in transmission rate reduces. This is due to the fact that the subcarriers are first arranged in sorted manner according to their SNR and subcarriers with maximum SNR are first allocated to the relay. From Figure 7 it is also observed that when the number of relays is less than the required number, then only subcarriers with maximum SNR are relayed through the available relays resulting in poor transmission rate. This confirms the need of multiple relays in CRN.

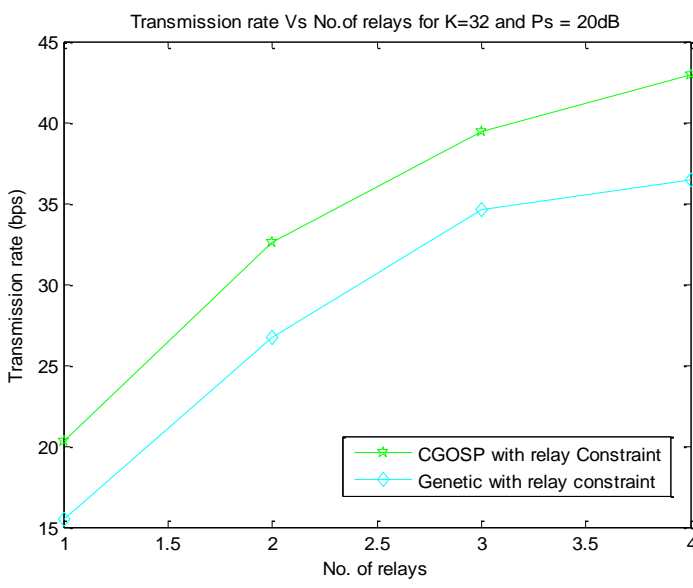

Figure 6. Transmission rate versus number of relays with $\mathrm{K}=32$, $\mathrm{Ps}=20 \mathrm{~dB}$, Pthresh $=-10 \mathrm{~dB}, \mathrm{M}=3$

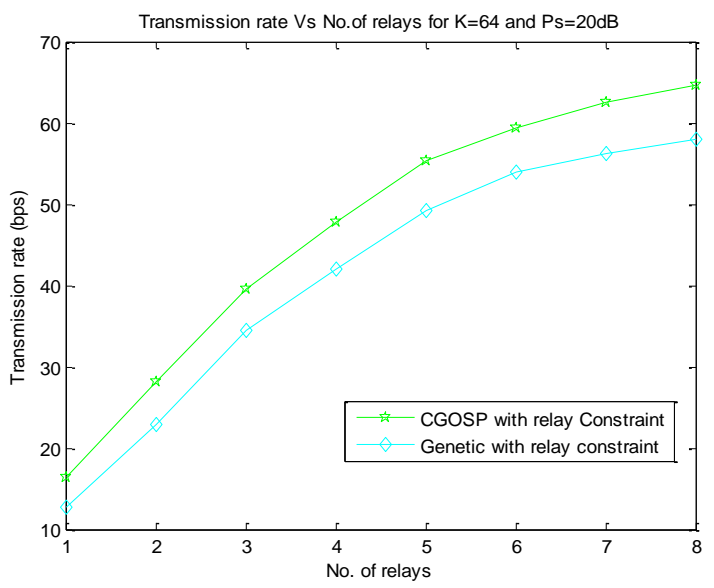

Figure 7. Transmission rate versus number of relays with $\mathrm{K}=64$, Ps $=20 \mathrm{~dB}, \mathrm{P}_{\text {thresh }}=-10 \mathrm{~dB}, \mathrm{M}=3$

Complexity for various algorithms are calculated and listed in Table 2. For GA, complexity due to power allocation is $\mathrm{O}$ (P1KI1) where $\mathrm{P} 1$ is the number of parent chromosomes generated in the first iteration, $\mathrm{K}$ is the number of subcarriers and $\mathrm{I} 1$ is the number of iterations required to obtain the optimized solution, and complexity for the subcarrier pairing is $\mathrm{O}(\mathrm{P} 2 \mathrm{KI} 2)$ where $\mathrm{P} 2$ and $\mathrm{I} 2$ are the number of parent chromosomes and number of iterations respectively. Hence, the combined complexity of the GA is $\mathrm{O}$ $(\mathrm{P} 1 \mathrm{KI} 1+\mathrm{P} 2 \mathrm{KI} 2)$. When we add multiple relays in the environment, complexity of the algorithm increases and it is $\mathrm{O}((\mathrm{P} 3 \mathrm{KI} 3+\mathrm{P} 4 \mathrm{KI} 4) \mathrm{N})$. In CGOSP algorithm, we are using OSP algorithm for subcarrier pairing part. 
Hence, the complexity of the algorithm decreases, and it is $\mathrm{O}\left(\mathrm{P}_{5} \mathrm{KI}_{5}+\mathrm{K}^{2}\right)$. However, when we apply relay constraint on it, complexity increases to $\mathrm{O}\left(\left(\mathrm{P}_{6} \mathrm{KI}_{6}+\mathrm{K}^{2}\right) \mathrm{N}\right)$. Then too this complexity is less than the complexity of the relay-constrained GA. Table 2 shows that CGOSP algorithm has almost half complexity compared to the GA.

Table 2. Complexity Comparison of Various Algorithms

\begin{tabular}{ll}
\hline \multicolumn{1}{c}{ Algorithms } & \multicolumn{1}{c}{ Complexity (No. of multiplications required ) } \\
\hline OSP [15] & $\mathrm{O}\left(\mathrm{K}^{2}\right)$ \\
GA (combined complexity) & $\mathrm{O}\left(\mathrm{P}_{1} \mathrm{KI}_{1}+\mathrm{P}_{2} \mathrm{KI}_{2}\right)$ \\
GA with relay constraint & $\left.\mathrm{O}\left(\mathrm{P}_{3} \mathrm{KI}_{3}+\mathrm{P}_{4} \mathrm{KI}_{4}\right) \mathrm{N}\right)$ \\
CGOSP algorithm & $\mathrm{O}\left(\mathrm{P}_{5} \mathrm{KI}_{5}+\mathrm{K}^{2}\right)$ \\
CGOSP algorithm with relay constraint & $\left.\mathrm{O}\left(\mathrm{P}_{6} \mathrm{KI}_{6}+\mathrm{K}^{2}\right) \mathrm{N}\right)$ \\
HGA [15] & $\mathrm{O}\left(\mathrm{K}^{2} \mathrm{I}_{7}+\mathrm{P}_{4} \mathrm{KI}_{7}\right)$ \\
Hungarian method [18] & $\mathrm{O}\left(\mathrm{K}^{2} I_{7}\right)$ \\
\hline
\end{tabular}

\subsection{Calculation of JFI}

Table 3 shows the values of JFI for various algorithms. $\mathbf{J}_{\min }$ is assumed as 0.8 (for long term) [16]. On an average, JFI for ordered allocation and Round Robin allocation is calculated. It is observed the overall range varies from 0.65 to 0.98 . For instance, for CGOSP JFI obtained for ordered allocation is 0.7 while for round Robin allocation it is .98. This proves that proposed algorithm which adopts Round Robin allocation allocates recourses with high degree of fairness which is very much essential for maintaining QoS among SUs.

Table 3. JFI calculation with $\mathrm{K}=32$, $\mathrm{Ps}=20 \mathrm{~dB}, \mathrm{M}=3, \mathrm{~N}=4, \mathrm{~L}=8$

\begin{tabular}{|c|c|c|c|c|c|c|c|}
\hline \multirow{2}{*}{ Algorithm } & \multirow{2}{*}{$\begin{array}{l}\text { Transmission } \\
\text { rate }\end{array}$} & \multicolumn{3}{|c|}{ Ordered allocation } & \multicolumn{3}{|c|}{ Round Robin allocation } \\
\hline & & $\mathrm{R}(1)$ & $\mathrm{R}(2)$ & JFI & $\mathrm{R}(1)$ & $\mathrm{R}(2)$ & JFI \\
\hline CGOSP & 44.1777 & 36.5229 & 7.6548 & 0.7 & 24.6129 & 19.5648 & 0.98 \\
\hline $\mathrm{GA}$ & 40.4973 & 34.2386 & 6.2587 & 0.67 & 23.2404 & 17.2569 & 0.97 \\
\hline
\end{tabular}

\section{CONCLUSION}

In this paper, an OFDM based cognitive multi relay network is investigated to improve the transmission rate of the cognitive radio (CR) with enhanced fairness among CR users with interference to the primary users (PUs) being managed below a predefined threshold level. In order to improve the transmission rate of the $\mathrm{CR}$, optimization of the subcarrier pairing and power allocation is carried out simultaneously. The joint optimization problem is formulated and a novel Composite Genetic and Ordered Subcarrier Pairing (CGOSP) algorithm is proposed to solve the problem and to reduce the complexity of Genetic Algorithm (GA). Further, to have a fair allocation of resources among CR users, the Round Robin allocation method is adopted so as to allocate subcarrier pairs to relays efficiently. The degree of fairness of the system is calculated using Jain's Fairness Index (JFI). Simulation results validate the significant improvement in transmission rate of the $\mathrm{CR}$, low computational complexity and enhanced fairness as compared to a similar state of the art work in the field.

\section{REFERENCES}

[1] S. Force, "Spectrum policy task force report," Federal Communications Commission ET Docket 02, vol. 135, 2002.

[2] J. Mitola and G. Q. Maquire, "Cognitive radio: Making software radios more personal," IEEE Pers. Commun., vol. 6, no. 4, pp. 13-18, 1999.

[3] A. S. Khobragade and R. D. Raut, "Hybrid Spectrum Sensing Method for Cognitive Radio," International Journal of Electrical and Computer Engineering, vol. 7, no. 5, 2017.

[4] C. S. Preetham, et al., "Performance Analysis of Cooperative Hybrid Cognitive Radio Network with Various Diversity Techniques," International Journal of Electrical and Computer Engineering, vol. 6, no. 5, pp. 2125-2133, 2016.

[5] H. V. Kumar, et al., "A Novel Approach to Optimize Cognitive Radio Network Utilization using Cascading Technique," TELKOMNIKA (Telecommunication Computing, Electronics and Control), vol. 13, no. 4, pp. 1233-1241, 2015.

[6] M. Shaat, et al., "Resource allocation in OFDM-based cognitive two-way multiple-relay networks," Proceedings of the 2014 IEEE Global Communications Conference, Austin, TX, pp. 917-922, 2014. 
[7] M. Wu, et al., "Power allocation for cognitive relay networks," Proceedings of the 2nd IEEE International Conference on Network Infrastructure and Digital Content 2010, Beijing, pp. 41-45, 2010.

[8] C. N. Hsu, et al., "Joint subcarrier pairing and power allocation for OFDM transmission with decode-and-forward relaying," IEEE Trans. Signal Processing, vol. 59, pp. 399-414, 2011.

[9] K. Xiong, et al., "Joint subcarrier-pairing and resource allocation for two-way multi-relay OFDM networks," Proceedings of the 2012 Global Communications Conference, Anaheim, CA, pp. 4874-79, 2012.

[10] Y. Li, et al., "Subcarrier pairing for amplify-and-forward and decode-and-forward OFDM relay links," IEEE Commun. Let., vol. 13, pp. 209-211, 2009.

[11] H. S. Lang, et al., "Joint subcarrier pairings and power allocations with interference management in cognitive relay networks based on genetic algorithms," Proceedings of the 2014 International Conference on Intelligent Green Building and Smart Grid, Taipei, pp. 1-5, 2014.

[12] L. Gao, et al., "Adaptive unbalanced spectrum usage and power allocation for cognitive OFDM-Based relay networks," Proceedings of the 5th International Conference on Wireless Communications, Networking and Mobile Computing, Beijing, pp. 1-4, 2009.

[13] G. Bansal, et al., "Subcarrier and Power Allocation for OFDMA-Based Cognitive Radio Systems with Joint Overlay and Underlay Spectrum Access Mechanism," IEEE Trans. Veh. Tech., vol. 62, pp. 1111-22, 2013.

[14] P. S. Varade and Y. Ravinder, "Optimal spectrum allocation in Cognitive Radio using Genetic Algorithm," Proceedings of the 2014 Annual IEEE India Conference, Pune, India, pp. 1-5, 2014.

[15] H. S. Lang, et al., "Subcarrier pairing and power allocation with interference management in cognitive relay networks based on genetic algorithms," IEEE Trans. Veh. Tech., vol. 65, pp. 7051-63, 2016.

[16] C. Guo, et al., "Throughput maximization with short-term and long-term Jain's index constraints in downlink OFDMA systems," IEEE Trans. Communications, vol. 62, pp. 1503-17, 2014.

[17] Holland and John H., "Genetic algorithms," Scientific american, vol. 267, pp. 66-72, 1992.

[18] X. Li, et al., "Joint power allocation andsubcarrier pairing for cooperative OFDM AF multi-relay networks," IEEE Commun. Lett., vol. 17, no. 5, pp. 872-875, 2013. 